\title{
ARQUEOLOGÍA EN EL PATIO DE LA FACULTAD DE HUMANIDADES Y ARTES. INFORME DE ACTIVIDADES TEMPORADA 2018
}

\author{
Diana S. Tamburini ${ }^{1}$, Mónica P. Valentini ${ }^{2}$, Mariana Algrain ${ }^{3}$, \\ Fernando Oliva ${ }^{4}$, Fausto Battaggia ${ }^{5}$, Guido Scaglione ${ }^{6}$, \\ Franco Abatangelo ${ }^{7}$ y Gabriel Spinetta ${ }^{8}$
}

Recibido: 15 de mayo de 2019. Aceptado: 20 de agosto de 2019

\section{Introducción}

En este Anexo se presenta un informe de las tareas de monitoreo y rescate llevadas a cabo en el patio de la Facultad de Humanidades y Artes, Universidad Nacional de Rosario. Desde el inicio del hundimiento de un sector del patio en octubre de 2018, la Facultad inició el recambio de los desagües ubicados en el patio. A partir de ello se realizará en el 2019 una reestructuración del patio con cambio de piso, renovación de pluviales y optimización de su uso para recreación. Desde entonces, un equipo de investigadores del Departamen- to de Arqueología integrado por docentes y alumnos de la misma facultad efectuó un monitoreo de la obra civil y diversas tareas de campo. Estas actividades contaron desde su inicio con el aval de las autoridades. Se articuló así, junto con el plan de obras, la puesta en valor del patio histórico con el fin de establecer su potencial valor arqueológico (Algrain et al. 2019) y de esta manera reinterpretar dentro de conjuntos teóricos amplios las diferentes ocupaciones que tuvo este emblemático lugar.

En el transcurso de las actividades de campo, se hallaron materiales correspondientes a fines del siglo XIX y principios

1 Departamento de Arqueología, Escuela de Antropología, Facultad de Humanidades y Artes. Universidad Nacional de Rosario. E-mail: dianatamburini@hotmail.com

2 Departamento de Arqueología, Escuela de Antropología, Facultad de Humanidades y Artes, Universidad Nacional de Rosario. E-mail: mopavalentini@gmail.com

3 Departamento de Arqueología, Escuela de Antropología, Facultad de Humanidades y Artes. Universidad Nacional de Rosario. E-mail: algrainmariana@hotmail.com

4 Departamento de Arqueología, Escuela de Antropología, Facultad de Humanidades y Artes. Universidad Nacional de Rosario. E-mail: fwpoliva@gmail.com

5 Departamento de Arqueología, Escuela de Antropología, Facultad de Humanidades y Artes. Universidad Nacional de Rosario. battaggia@hotmail.com

6 Departamento de Arqueología, Escuela de Antropología, Facultad de Humanidades y Artes. Universidad Nacional de Rosario. E-mail: guidoscaglione94@gmail.com

7 Departamento de Arqueología, Escuela de Antropología, Facultad de Humanidades y Artes. Universidad Nacional de Rosario. E-mail: franciabatangelo@gmail.com

8 Departamento de Arqueología, Escuela de Antropología, Facultad de Humanidades y Artes. Universidad Nacional de Rosario. E-mail: gabrielspinetta@hotmail.com 
del siglo XX (gres cerámico, loza, vidrio, metal). Estos últimos posiblemente venian en el sedimento del relleno utilizado. Se trata de elementos que formaron parte del relleno efectuado en el anterior reemplazo de conductos de desagües. Junto a estos materiales, sorprende el hallazgo de pequeños y medianos fragmentos de cerámica prehispánica. Algunos de estos fragmentos presentan un siglado particular y tal vez tengan relación con los depósitos de las colecciones arqueológicas que estaban en el Instituto de Antropología (ver González 1959), producto de los materiales que se traían de las primeras excavaciones arqueológicas que los miembros del Instituto realizaban y que por diferentes razones que se están analizando terminaron en esos desagües.

\section{Breve reseña histórica}

El edificio de la hoy Facultad de Humanidades y Artes de la Universidad Nacional de Rosario (ubicado en la calle Entre Ríos 758) (Figura 1) fue proyectado por el arquitecto británico Arthur Inglis en 1902 y construido por el arquitecto italiano Felipe Censi. Fue creado para el colegio de estudiantes externas de la orden religiosa que se instalara en la ciudad a fines del siglo XIX, La Santa Unión de los Sagrados Corazones (Figura 2).

Este inmueble de planta baja y dos pisos se ubicó en un gran lote medianero de forma irregular (Figura 3). Está organizado según el esquema del claustro, en que las aulas rodean un patio central de importantes dimensiones. Hoy es un patio seco (Figura 4), pero que en la época de las religiosas podría haber tenido incluso una huerta, como era costumbre. Recostado sobre la medianera sur se estableció el sector privado de la orden, localizando la capilla en el primer piso sobre el mismo sector, en la actualidad en este espacio funciona la biblioteca central de la facultad (Guía de Arquitectura de Rosario 2003).

Arquitectónicamente el edificio, de líneas asimétricas y austeras, se enmarca en el estilo neogótico inglés, respondiendo al eclecticismo academicista instaurado en la época. El 9 de agosto de 1947, fue creada la Facultad de Filosofia, Letras y Ciencias de la Educación de la Universidad Nacional del Litoral, que comenzó a funcionar a partir del 5 de junio de 1948 en el recinto del Colegio Nacional $\mathrm{N}^{\circ} 1$. E1 29 de diciembre de 1951 el Consejo Superior decidió la compra del actual edificio, en el que funcionaba el colegio de La Santa Unión que se trasladó a su predio original de las calles Salta y Ovidio Lagos (https:// fhumyar.unr.edu.ar/ ). En 1952, por decreto del Poder Ejecutivo de la Nación se lo declaró de utilidad pública y sujeto a expropiación con destino a la Facultad de Filosofia y Letras y otras secciones de la Universidad Nacional del Litoral (http://rosariociudad.blogspot.com.ar/). En una fotografia publicada por el Diario La Capital de Rosario con fecha 2 de enero de 1955, se distinguen en el patio de la Facultad canteros paralelos a los muros del edificio. Estas construcciones desaparecieron en una reforma posterior (Figura 5).

El 29 de noviembre de 1968, el Gobierno Nacional dictó la ley $17.987 / 68$, en virtud de la cual, y sobre la base de siete Facultades, Institutos y Organismos dependientes de la Universidad Nacional del Litoral, se creó la décima universidad nacional argentina: la Universidad Nacional de Rosario. Entre esas siete facultades estaba la de Filosofia y Letras que, adquirió su nombre actual el 15 de junio de 1979 (e-universitas.edu.ar/jour_store/documentos/historia_esp.pdf).

Lamentablemente, no se cuenta con los planos originales que testimonien cómo era el edificio en su interior ni los cambios que sufrió a través del tiempo. Las entrevistas realizadas a quienes trabajaron en 


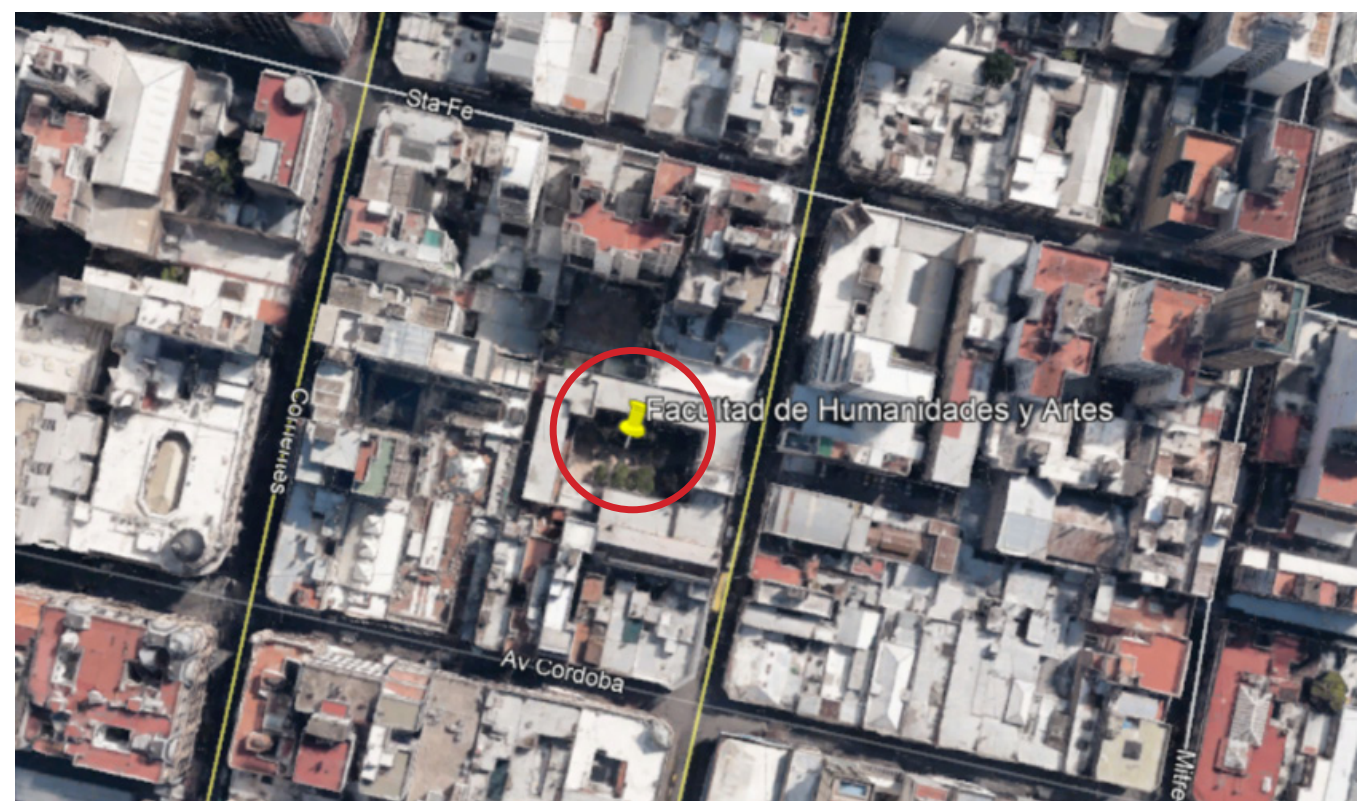

Figura 1. Imagen satelital de la actual Facultad de Humanidades y Artes, UNR (Fuente Google Earth 2019).
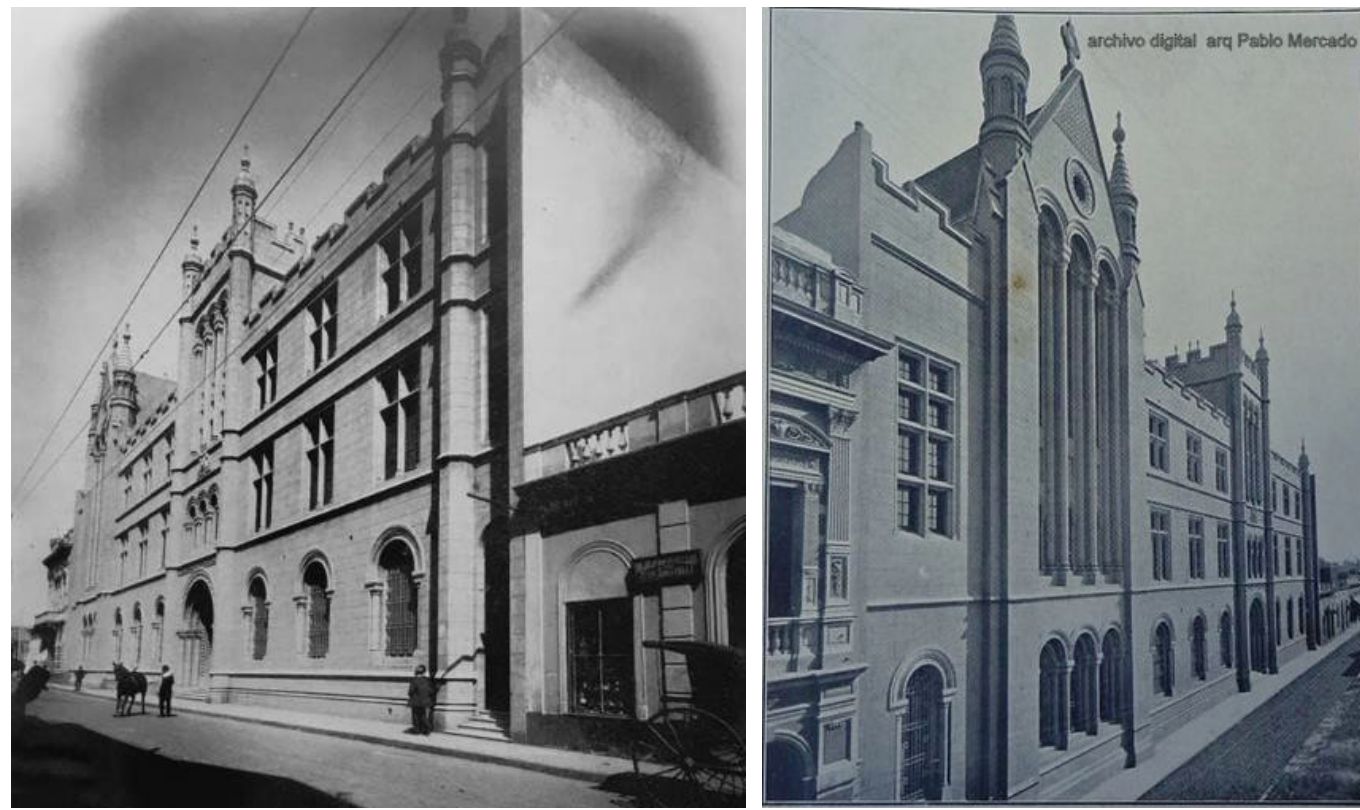

Figura 2. Fotografias de 1905 y 1910 respectivamente del edificio de la actual Facultad de Humanidades y Artes. Archivo digital Arquitecto Pablo Mercado. 
Figura 3. Plano de Catastro Municipal. Dirección General de Topografia y Catastro. Municipalidad de Rosario. Gentileza Arquitecta María Virginia Marull.

Figura 4. Vista del patio desde el $2^{\circ}$ piso hacia el oeste (2018).
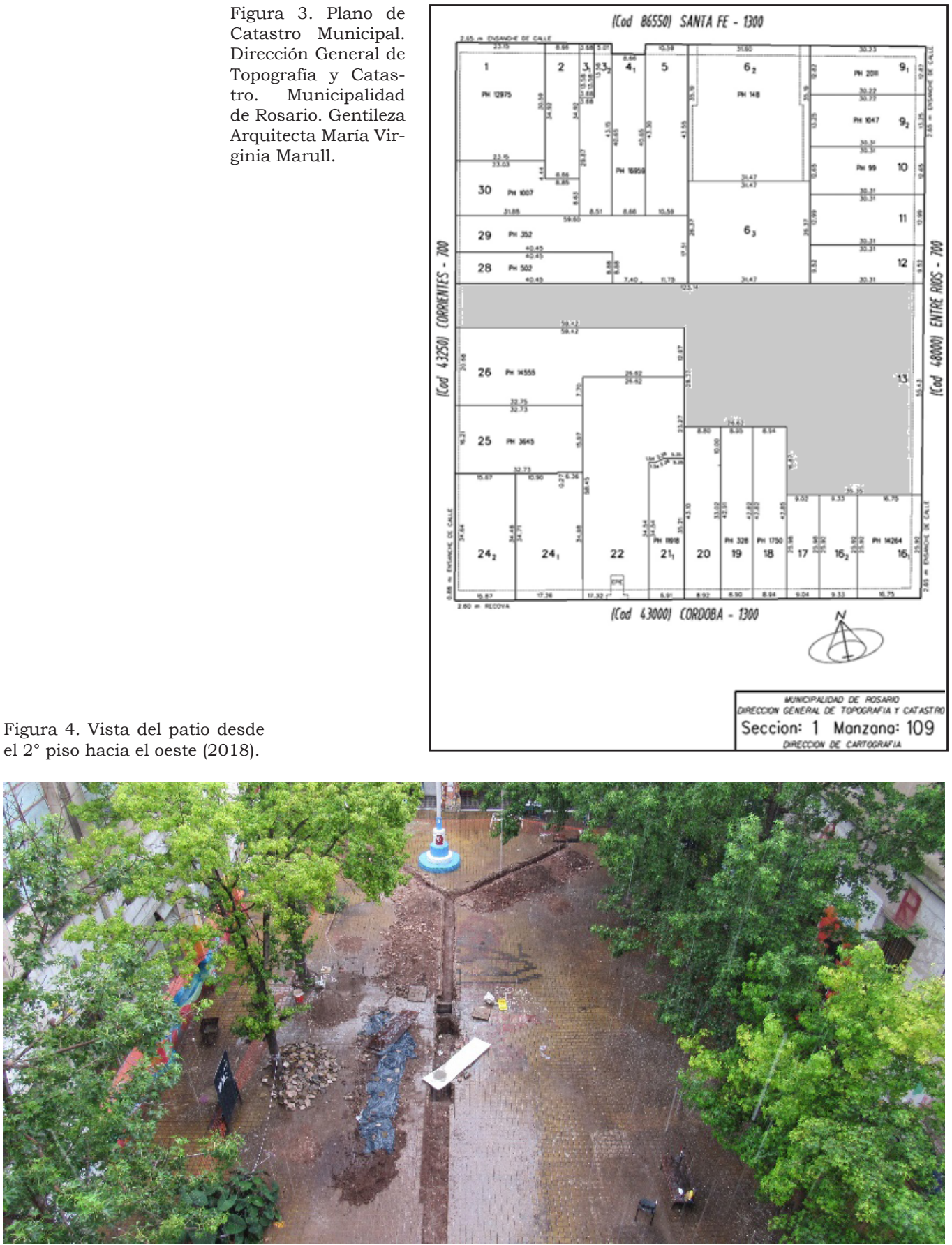
la facultad hace ya 40 años, recuerdan el patio en la situación actual. Sólo se conserva en el Programa de Preservación Documental, Investigación, Formación y Extensión: "La Facultad de Humanidades y Artes Memoria Historia y Politica", una tira de prueba fotográfica de finales de la década de 1960, en la cual se distingue la presencia del mástil en el patio de la Facultad en medio de una posible reforma (Figura 6).

\section{Metodologia}

Con el objetivo de profundizar el conocimiento disponible acerca de la historia de la Facultad se llevaron a cabo las siguientes tareas:

- Relevamiento documental (catastro; biblioteca central de la Facultad; archivo documental)

- Entrevistas a personal no docente jubilados (Pedro Andrisani) y graduados (Soccorso Volpe).

- Recuperación de materiales, registro y análisis de estos.

- Difusión de los resultados (Primeras Jornadas de Práctica Arqueológica, julio 2019).

A raíz de las obras de restauración del patio histórico de la Facultad, el monitoreo de la obra civil (Figura 7) permitió distinguir elementos constructivos relacionados con rellenos, compactaciones, bases de columnas y posibles pisos anteriores al inmueble actual, tal vez anterior al edificio perteneciente a la congregación de las religiosas.

En esta etapa, donde se renovaron cañerias, se realizaron tareas arqueológicas de seguimiento de obra, mapeo de perfiles, zarandeo de lo removido y recuperación in situ (Figuras 8, 9 y 10). En un primer momento se distinguen en la zanja abierta por los obreros para la ejecución de la obra, debajo del piso bases de $50 \mathrm{~cm}$ de ancho y 60

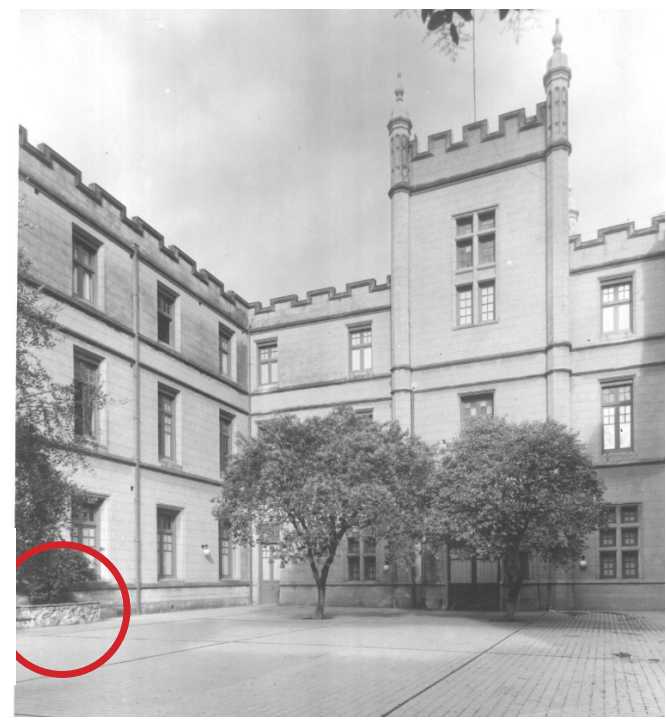

Figura 5. Vista del patio desde el oste. Diario La Capital de Rosario, Santa Fe. Año 1955 (colección privada)

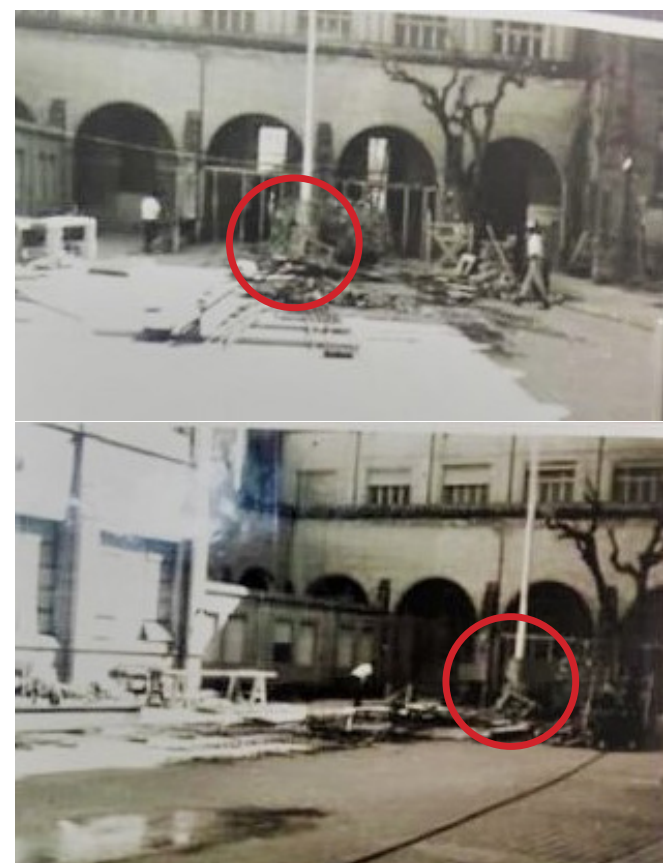

Figura 6. Ubicación del Mástil en la década de 1960. (Gentileza Doctora Cristina Viano. Programa de Preservación Documental, Escuela de Historia, FHyA). 


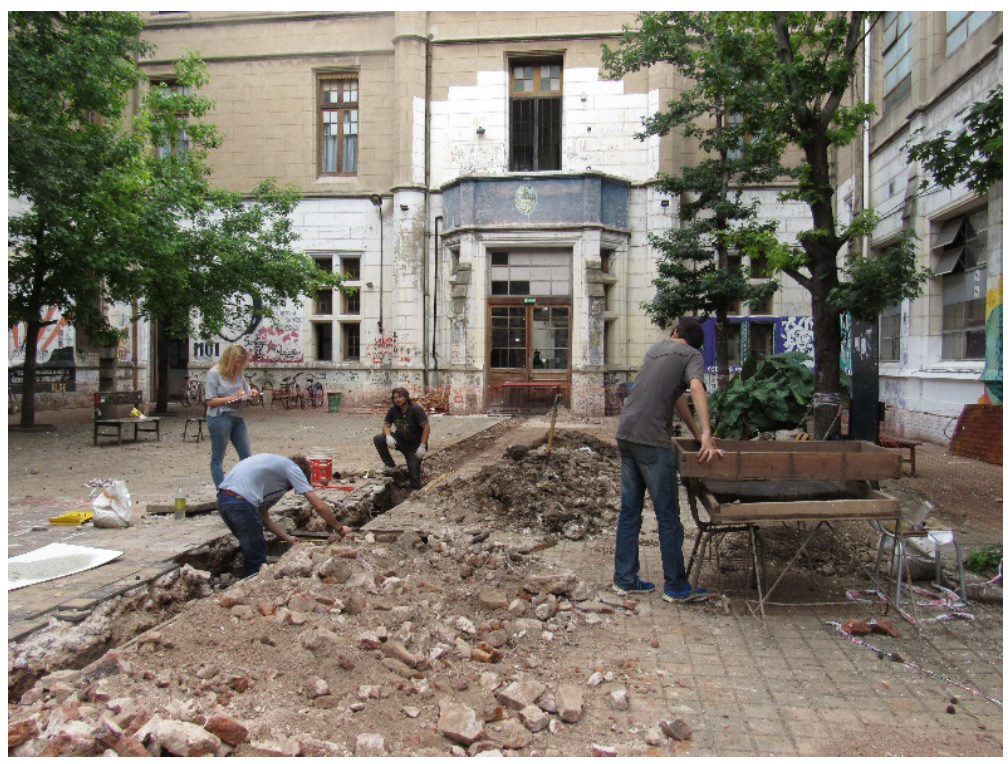

Figura 7. Monitoreo de la zanja abierta por los obreros (vista desde el oeste, 2018).

de profundidad, que pueden corresponder a pilares de mampostería pertenecientes a una edificación anterior. Junto al material removido como al material expuesto, se recuperan materiales del periodo histórico y arqueológico entre los sedimentos extraídos con zarandas de malla gruesa y mediana, sectorizando las muestras.

El acervo arqueológico reunido hasta el momento ya aporta identificaciones comunes con relación a ciertos rasgos arqueológicos registrados en el subsuelo de edificios históricos. Algunos de estos rasgos pueden ser distinguidos como efectivos patrones arqueológicos para un determinado período. La zanja abierta para el recambio de conductos posibilitó distinguir la presencia de una capa de relleno, este sedimento artificial utilizado para nivelar la superficie y preparado como base para la construcción de un nuevo piso puede considerarse como un rasgo arqueológico.

En líneas generales este relleno incluye tanto escombros y materiales cerámicos de construcción como ser fragmentos de cañerías de cerámica vitrificada, ladrillos ${ }^{1}$ y restos de basura. ${ }^{2}$ Como así también bases de botellas de vidrio de sección redonda color negro, azulejos, un borde de loza de plato pequeño, vidrio meteorizado, un recipiente circular de hierro, metales varios ${ }^{3}$ y varios fragmentos de botellas de gres de cerveza entre los que sobresalen uno color blanco con el sello de la fábrica y otro bicolor ${ }^{4}$ (Figura 11).

Se destaca la presencia de cerámica prehispánica siglada (Figura 12) la importancia del hallazgo de fragmentos de cerámica indígena se relaciona posiblemente, como ya se mencionó, con los depósitos de materiales arqueológicos que estaban en el Instituto de Antropología, ubicado en el 2do piso de la Facultad, y que, por diferentes razones que se están analizando, terminaron en esos desagües. Es probable que se corresponda con alguna de las colecciones fundacionales pertenecientes al Instituto aunque las siglas de los tiestos no 


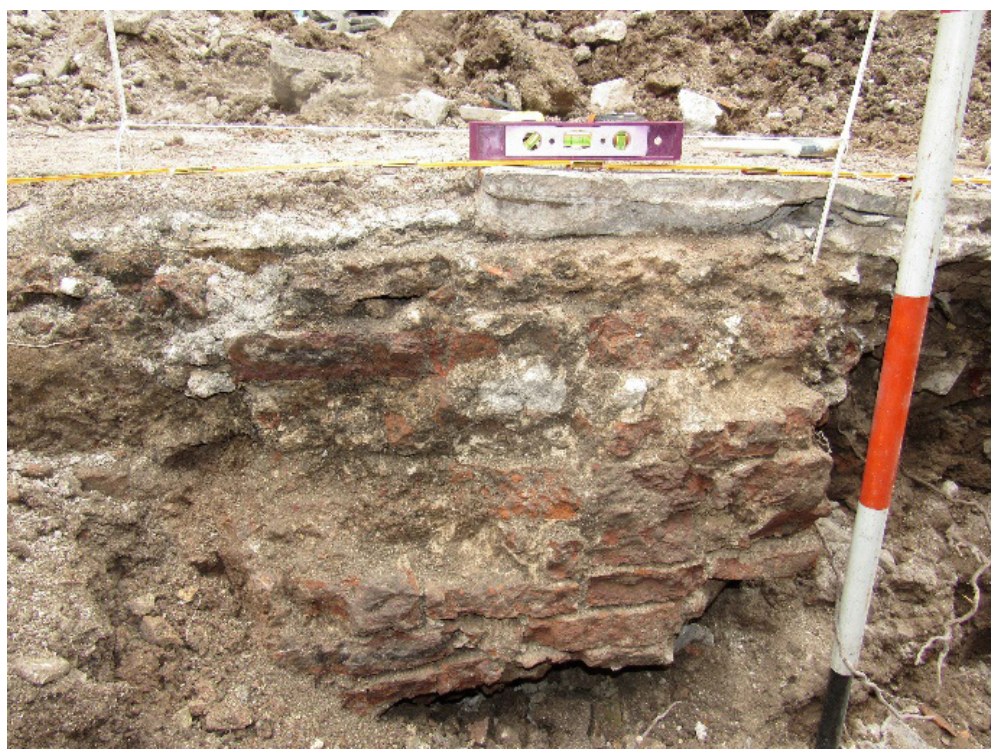

Figura 8. Perfil estructura sur (2018).
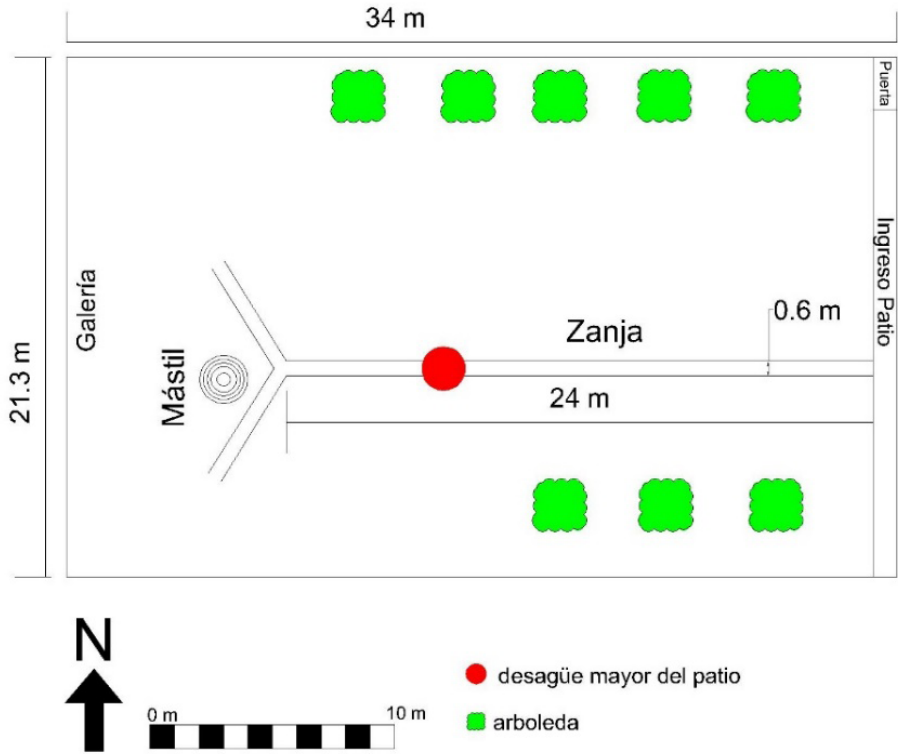

Figura 9. Croquis del patio con la zanja realizada por los obreros (dibujo Juan Cruz Aramburu). 


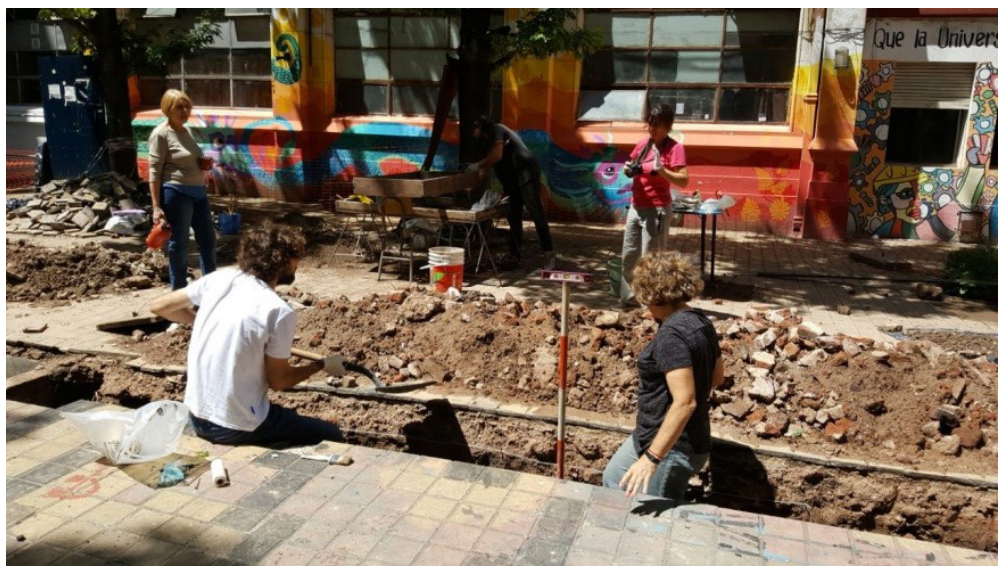

Figura 10.

Registro del perfil (2018).

se corresponden con el inventario de este.

Un relato oral imperante en la Facultad da cuenta de la destrucción de las colecciones arqueológicas fundacionales del Instituto de Antropología, que ocurrió durante la última dictadura cívico-militar, (denominada por los militares como Proceso de Reorganización Nacional a partir del golpe de Estado del 24 de marzo de 1976). Durante este período se produce no sólo el cierre de la carrera de Antropología, la persecución y desaparición física tanto de estudiantes como docentes universitarios, sino también, la destrucción y pérdida del valioso patrimonio arqueológico. Este relato afirma que los militares podrian haber destruido muchas de las piezas resguardadas en el Instituto al arrojarlas desde el segundo piso al patio histórico de la $\mathrm{Fa}$ cultad. Es por eso que una de las hipótesis que se maneja, es la posibilidad de que estas piezas de cerámica hayan formado parte de la colección antes de 1976. Pero el tamaño de los fragmentos no se corresponde con tiestos que logren ser remontados, incluso varios están erosionados y son de diferente espesor y colores. De acuerdo con la entrevista realizada a Soccorso Volpe, es
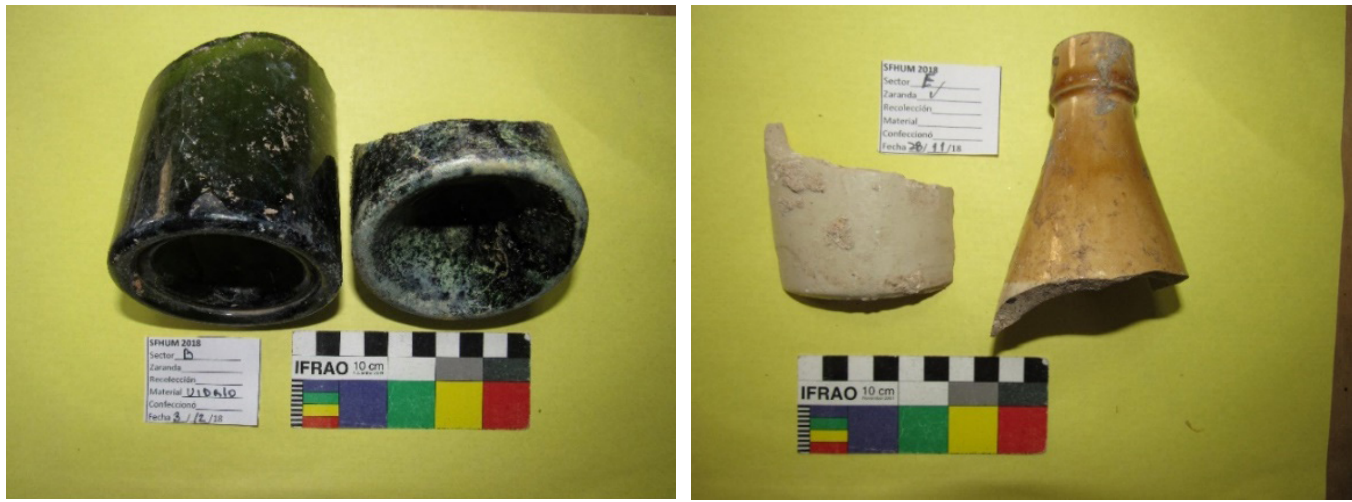

Figura 11. Fragmentos de vidrio negro y gres cerámico. 

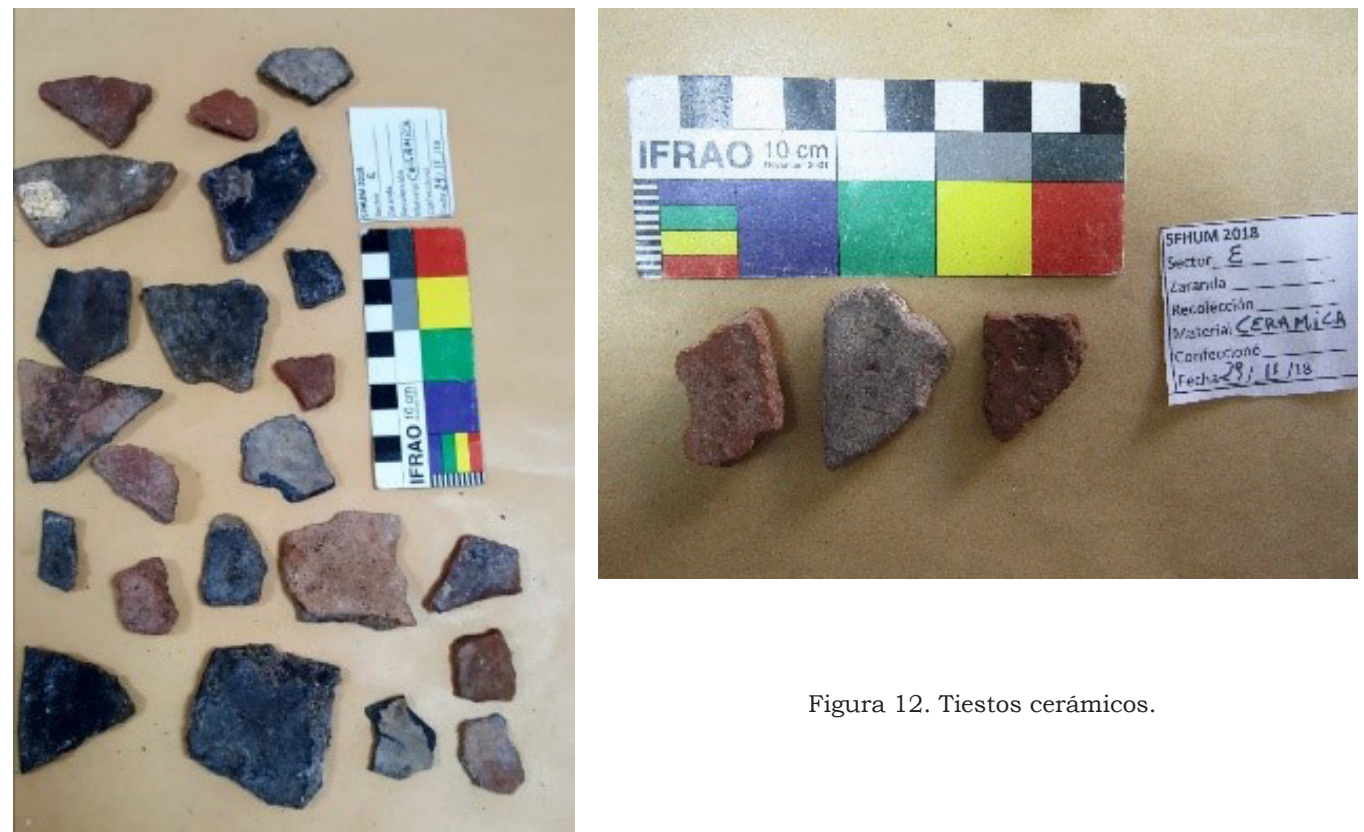

posible que tiraran por las ventanas del segundo piso algunos fragmentos cerámicos, pero no piezas enteras (comunicación personal, Volpe 2019).

\section{Consideraciones finales}

Este trabajo representa un informe extendido de las acciones realizadas hasta el momento, y a medida que avancen las obras de remodelación del patio histórico se continuará con el monitoreo correspondiente y, de ser necesario, se efectuará una excavación posicional. Asimismo, los materiales están siendo analizados en laboratorio para su descripción, identificación, interpretación y clasificación. A medida que avance el análisis de los materiales recuperados se intentará determinar el origen de los tiestos hallados en las excavaciones, así como establecer nuevas líneas de investigación. De esta manera, se espera profundizar el conocimiento acerca de la historia de la Facultad a través las investigaciones arqueológicas y el análisis de los materiales recuperados en el patio, poniendo en valor su pasado histórico. Es así como gracias a la investigación arqueológica se pueden reconocer diferentes funciones para un mismo inmueble en diferentes momentos de la historia, como es el caso de nuestra facultad.

\section{Agradecimientos}

A las autoridades de la FHyA. A la profesora Nélida De Grandis y a Lara por colaborar en las actividades de lavado de material, al Sr. Pedro Andrisani y al Lic. Soccorso Volpe por brindarnos información sobre la historia de la Facultad. A Alfredo Celoria por las fotografias y por último al Sr. Enzo Navas. 


\section{Notas}

1. Los mismos serán enviados al LEMIT (Laboratorio de Entrenamiento Multidisciplinario para la Investigación Tecnológica) para su caracterización.

2. Plásticos y material orgánico como huesos de animales, etc.

3. Esto incluye alambres, clavos, latas y fragmentos de hierro.

4. Estos contextos también demuestran la existencia de botellas de gres importadas y utilizadas como contenedores de cerveza $\mathrm{u}$ otros líquidos, las cuales son similares a los ejemplares producidos y distribuidos por Holanda e Inglaterra en el siglo XIX (Schávelzon 1991, 1995 y 1996; Volpe 1994).

\section{Bibliografia}

AA.VV. 2003. Guía de Arquitectura de Rosario. Editorial Junta de Andalucía, España.

ALGRAIN, M.; TAMBURINI, D. S.; VALENTINI, M. y OLIVA, F. 2019. El pozo del patio: arqueología reciente en la Facultad de Humanidades y Artes, UNR. Libro de Resúmenes Primeras Jornadas de Práctica Arqueológica. Facultad de Humanidades y Artes, Universidad $\mathrm{Na}$ cional de Rosario.

GONZALEZ, A. R. 1959. Prólogo. En Revista del Instituto de Antropología. Facultad de Filosofia y Letras. UNL. Tomo 1, pp 5 a 7.

SCHÁVELZON, D. 1991. Arqueología histórica de Buenos Aires (I): la cultura material porteña de los siglos XVII y XIX. Editorial Corregidor, Buenos Aires.

SCHÁVELZON, D. 1995. Arqueología histórica de Buenos Aires (III): la Imprenta Coni. Editorial Corregidor, Buenos Aires.

SCHÁVELZON, D. 1996. Catálogo de cerámicas históricas del Río de la Plata (siglos XVI-XX), apéndice gráfico. Centro de Arqueología Urbana, Buenos Aires
VOLPE, S. 1994. Tipología de recipientes de gres cerámico y precintos de cerveza, excavaciones arqueológicas de Rosario. Publicación Nro. 19, Centro de Arqueología Urbana, Buenos Aires.

\section{Fuentes on line:}

https://fhumyar.unr.edu.ar/ (consultada el 18/02/2019)

http: / / rosariociudad.blogspot.com.ar (consultada 12/03/2019)

e-universitas.edu.ar/jour_store/documentos/historia_esp.pdf(consultada12/03/2019) 